\title{
(2) Bibliocanto?
}

PLATAFORMA PAPAYA: DESAFIOS NA IMPLEMENTAÇÃO DE

PAPAYA PLATFORM: IMPLEMENTATION CHALLENGES ON DIGITAL LIBRARIES AND ARCHIVES

Roberto Fray da Silva ${ }^{1}$

roberto.fray.silva@gmail.com

Fernando Hattori ${ }^{2}$

hattori@usp.br

Carlos Alberto Agarie Junior ${ }^{3}$

carlos.agarie@gmail.com

Kollontai Cossich Diniz ${ }^{4}$

tai@zeromail.org

Edson Satoshi Gomi ${ }^{5}$

gomi@usp.br

Resumo: A digitalização de acervos é importante tanto como ferramenta para a ampliação do alcance de documentos quanto para a conservação destes. A implementação dos repositórios que receberão esses acervos não é simples e apresenta particularidades que dependem do contexto do acervo e

\footnotetext{
${ }^{1}$ Bacharel em Engenharia Agronômica - USP. Mestre em Engenharia de Sistemas Logísticos USP. Doutor em Engenharia Elétrica - USP. Pesquisador - LLA. Lattes: http://lattes.cnpq.br/8941366680536142.

${ }^{2}$ Bacharel em Sistemas de Informação - USP. Mestre em Engenharia da Computação - USP. Lattes:

${ }^{3}$ Bacharel em Engenharia de Automação e Controle - USP. Mestrando em Aprendizagem de Máquina - USP. Lattes: http://lattes.cnpq.br/3273976822629093

${ }^{4}$ Bacharel em Desenho Industrial - UnB. Mestre em Arquitetura e Urbanismo - USP. Pesquisador - CEBRAP. Lattes: http://lattes.cnpq.br/5819080126645250.

${ }^{5}$ Bacharel em Engenharia Elétrica - EPUSP. Mestre em Engenharia Elétrica - EPUSP. Doutor em Engenharia Eletrônica - TODAI, Japão. Professor Doutor do Departamento de Engenharia de Computação e Sistemas Digitais - USP. Lattes: http://lattes.cnpq.br/1621866499641636.
} 


\section{(2Bibliocanto@}

106

dos seus procedimentos operacionais. A Plataforma Papaya é uma ferramenta para o gerenciamento de acervos digitais. Neste trabalho são apresentadas quatro implementações desta plataforma, duas em acervos de bibliotecas e duas em acervos de arquivos, com seus respectivos requisitos e as decisões de projeto tomadas durante o desenvolvimento.

Palavras-chave: Acervos (bibliotecas). Acesso à informação. Arquivos (instituição). Programas de código aberto. Bibliotecas digitais.

\section{INTRODUÇÃO}

A digitalização de acervos históricos vem aumentando de importância nos últimos anos (MARTINS, RODRIGUES, NUNES, 2008; LYNCH, 2003; LESK, 1995) devido a um conjunto de fatores: melhores procedimentos e equipamentos para captura de imagens, software para processamento de imagens com maior facilidade de uso, desenvolvimento de software para criar e gerenciar repositórios digitais, elaboração de padrões para catalogação de documentos digitais, dentre outros. Esta pode ser dividida em duas classes: digitalização em massa, que lida com a geração de documentos digitais com base em documentos físicos em larga escala, buscando padronização, e digitalização tradicional, que visa produzir documentos de maior qualidade, normalmente em projetos de preservação digital (COYLE, 2006).

No Brasil, existem repositórios digitais de diversos tipos: bibliotecas, museus, arquivos históricos, documentos empresariais, documentos oficiais, teses e dissertações, dentre outros. Um levantamento realizado pelo Instituto Brasileiro de Informação em Ciência em Tecnologia (IBICT, 2016) identificou 109 repositórios digitais no país. Dentre estes, alguns merecem destaque devido tanto à quantidade de documentos quanto à sua diversidade: Alice, o repositório de informações científicas da EMBRAPA (EMBRAPA, 2016), o Acervo 


\section{BiblioCanto?}

107

Digital do INMETRO (INMETRO, 2016), a Biblioteca Digital da Produção Intelectual da Universidade de São Paulo (USP, 2016), e a Biblioteca Multimídia da FIOCRUZ (FIOCRUZ, 2016).

Os principais requisitos que levaram ao desenvolvimento da Plataforma Papaya para acervos digitais foram: exibição de conteúdo no navegador (sem a necessidade de realizar o download do documento completo para visualizá-lo), uma interface mais intuitiva para os usuários finais, e a possibilidade de exibição em diferentes meios (desktops, smartphones e tablets) (PLATAFORMA PAPAYA, 2016).

O objetivo deste trabalho é apresentar os resultados de quatro projetos de implementação de repositórios digitais que utilizaram a Plataforma Papaya: dois relacionados a bibliotecas e dois relacionados a arquivos históricos. Todos os projetos já estão em funcionamento e podem ser consultados. Os principais problemas observados na implementação de cada projeto também serão citados, visando auxiliar o planejamento de outros grupos e instituições que se interessem por implementar um repositório digital.

\section{DESENVOLVIMENTO DE REPOSITÓRIOS DIGITAIS COM A PLATAFORMA PAPAYA}

A metodologia utilizada neste trabalho pode ser dividida em quatro etapas:

i. Levantamento de requisitos de gerenciadores de conteúdo e usuários na implementação de repositórios digitais;

ii. Customização da plataforma, envolvendo desenho de sua arquitetura, seleção de software e testes;

iii. Implementação da plataforma em dois projetos de criação de repositórios 


\section{Bibliocanto}

digitais em bibliotecas e dois projetos em arquivos históricos;

iv. Avaliação dos resultados obtidos e identificação de principais erros durante a implementação.

\subsection{LEVANTAMENTO DE REQUISITOS}

Durante uma série de reuniões com gerenciadores de conteúdo (bibliotecários e arquivistas, principalmente) e usuários finais (tanto técnicos quanto leigos no material da biblioteca ou arquivo em questão), foram definidos três categorias de requisitos: acesso, facilidade de uso e qualidade dos dados.

A primeira categoria contempla os seguintes requisitos: acesso livre à informação (visualização dos documentos inseridos na plataforma sem a necessidade de realizar login); uso de ferramentas de código livre $(100 \%$ do software e dependências deveriam possuir código livre); possibilidade de adicionar links para páginas externas relacionadas ao projeto em questão; acessibilidade em desktops, tablets e smartphones (compatibilidade com diferentes navegadores e sistemas operacionais).

A segunda categoria compreende os seguintes requisitos: busca facetada (deve ser possível realizar a busca em todos os campos de metadados); possibilidade de visualizar o conteúdo online (visualizadores para imagens, mapas, plantas e textos); usabilidade na interface do usuário final (ambiente considerado intuitivo por usuários finais); usabilidade na interface dos bibliotecários e arquivistas (ambiente considerado intuitivo pelos gerenciadores de conteúdo); facilidade de treinamento dos gerenciadores de conteúdo (máximo 1 dia de treinamento para utilizar a ferramenta); facilidade na inserção e gerenciamento de documentos (avaliada pelo número de problemas 


\section{Bibliocanto}

109

relatado por bibliotecários e arquivistas); e não realizar modificações na forma como os documentos são catalogados atualmente.

A terceira categoria é relacionada aos seguintes requisitos: busca sem erros nos campos de metadados (avaliada pelo número de erros relatados por usuários finais); e busca consistente e eficaz (avaliada pelo número de erros relacionados por usuários finais e gerenciadores de conteúdo).

\subsection{DESENVOLVIMENTO DAPLATAFORMA}

Para a elaboração da Plataforma Papaya, foram analisados os diferentes softwares de código livre que poderiam ser utilizados. Os principais fatores analisados foram: disponibilidade de uma grande quantidade de instalações em repositórios digitais; existência de uma comunidade ativa responsável pela manutenção do software; compatibilidade com diferentes padrões de metadados; e possibilidade de trabalhar com uma grande quantidade de arquivos. Após realizar a pesquisa das alternativas disponíveis, optou-se por utilizar o software DSpace (DSPACE, 2016). A plataforma Papaya possui a estrutura ilustrada na Figura 1. 


\section{Bibliocanto}

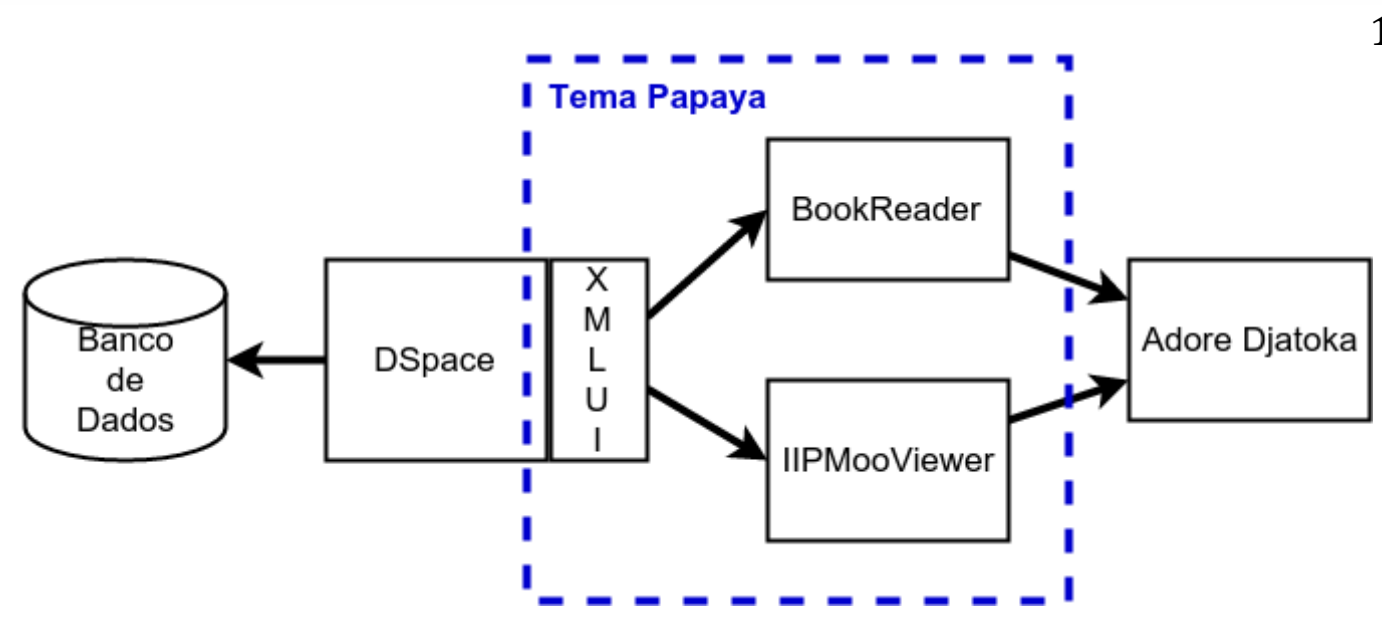

Figura 1 - Estrutura da Plataforma Papaya, com destaque para o tema Papaya.

Fonte: elaborado pelos autores, 2016.

O DSpace pode ser definido como uma aplicação de software livre para o gerenciamento e disponibilização online de acervos digitais (TANSLEY et. al., 2003). Este possui duas interfaces de interação com os usuários, uma denominada JSPUI, baseada em JavaServer Pages, e outra denominada XMLUI, desenvolvida com base no framework do Apache Cocoon (APACHE COCOON, 2016). Nessas interfaces, os responsáveis pelo acervo podem gerenciá-lo (inserir, modificar e deletar itens no repositório) e os usuários podem visualizar o acervo.

Uma nova interface gráfica foi desenvolvida para a Plataforma Papaya sobre o tema padrão já existente na interface XMLUI do Dspace (PHILLIPS et al., 2007), gerando um Tema Papaya. Esta nova interface foi desenvolvida para ser flexível e se adaptar a diferentes tamanhos de display, tornando a plataforma mais amigável em telas pequenas como aos presentes em dispositivos móveis. Além disso, foi adotada uma interface mais minimalista que facilita a navegação, removendo o excesso de informações presentes na página inicialmente. 


\section{(2Bibliocanto@}

$\mathrm{Na}$ página de busca, cada item é caracterizado por alguns poucos metadados (título, autor, ano e resumo) tornando a interface menos sobrecarregada, mas as informações de todos os metadados estão disponíveis em uma seção expedível que pode ser visualizada utilizando um botão logo abaixo do item.

Na plataforma desenvolvida, as páginas dos itens do acervo permitem a sua visualização rápida no próprio navegador sem a necessidade do usuário baixar nenhuma ferramenta externa, permitindo que o usuário visualize o item em qualquer navegador e julgue sua relevância antes de baixar o item completo do acervo. Além disso, essa visualização não depende de tecnologia Flash (ADOBE, 2016) e é amigável mesmo em ambiente móvel.

A visualização de livros é feita utilizando a ferramenta BookReader (INTERNET ARCHIVE, 2015). Esta ferramenta foi desenvolvida utilizando a linguagem javascript pelo Internet Archive com o objetivo de facilitar a disponibilização online de livros digitalizados. As páginas dos livros são carregadas na plataforma como imagens separadas. Desse modo, a ferramenta BookReader acessa cada uma dessas imagens e as exibe em formato de livro.

Já a visualização de imagens é feita utilizando a ferramenta IIPMooViewer (IIP IMAGE, 2015). Esta ferramenta foi desenvolvida utilizando a linguagem javascript também com o objetivo de permitir a visualização de grandes imagens. Este visualizador permite a exibição de uma única grande imagem e monta essa imagem utilizando um mosaico de imagens pequenas, desse modo, o servidor e os computadores dos usuários não serão sobrecarregados pelo download de arquivos muito grandes. $\mathrm{O}$ uso dessa ferramenta de visualização de grandes imagens é possível por causa do servidor de imagens Adore Djatoka (ADORE DJATOKA, 2013), que é capaz de servir eficientemente partes menores de uma imagem grande. Além disso, este 


\section{(2)Bibliocanto}

visualizador também possui uma pequena miniatura da imagem na qual ele exibe qual seção exata da imagem está sendo exibida e permite uma rápida navegação pela imagem.

O visualizador de grandes imagens foi modificado para permitir a exibição de mais de uma imagem por item do acervo. Ou seja, itens que possuem mais de 1 imagem (por exemplo, fotos ou mapas que possuem informações relevantes em seu verso) podem ser exibidos utilizando o mesmo visualizador de grandes imagens. A ferramenta IIPMooViewer permite a exibição de uma imagem de cada vez, mas a nova funcionalidade permite que sejam exibidas várias imagens e é possível alternar entre essas imagens utilizando botões localizados na lateral da interface.

O Adore Djatoka (ADORE DJATOKA, 2013) é um servidor de imagens, responsável na plataforma por servir as imagens em resolução e tamanhos ótimos para a interface de acordo com o tipo de visualização e o tamanho de tela utilizados. O Djatoka permite o uso de um visualizador de grandes imagens que não carrega toda a imagem em um único download.

\subsection{IMPLEMENTAÇÃO EM BIBLIOTECAS DIGITAIS}

A Plataforma Papaya encontra-se atualmente implementada em duas bibliotecas: a de documentos históricos da Escola Politécnica da Universidade de São Paulo (MEMORIA DA POLI, 2016) e o acervo do Departamento de Engenharia de Estruturas e Geotécnica (PEF, 2016). A Figura 2 contém a página inicial do repositório digital do primeiro projeto e a Figura 3 contém os resultados da busca "concreto" no repositório digital do segundo projeto. É importante notar, na Figura 3, a implementação dos filtros para facilitar a busca pelo usuário final. Estes podem ser definidos pelos administradores do repositório digital, porém observou-se que, no caso de bibliotecas, os campos 


\section{(2) Bibliocanto?}

de metadados mais importantes são: autor, assunto, coleção pertencente no repositório digital em questão e data de publicação do documento.

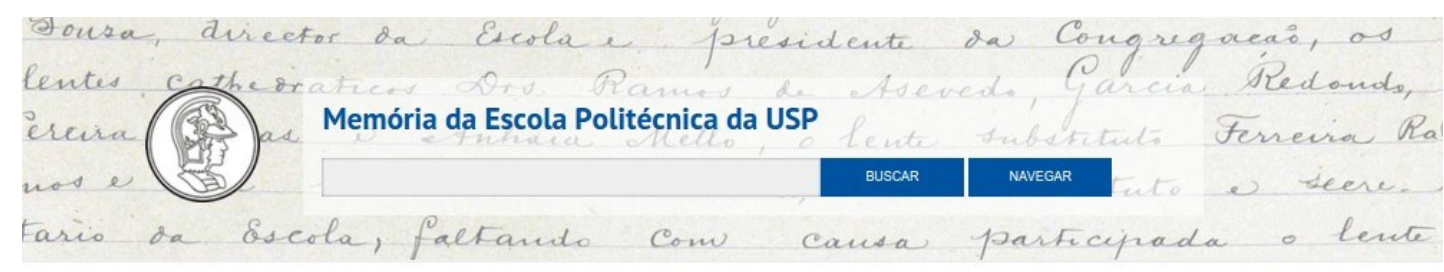

Coleçōes

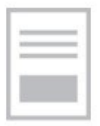

Anuários da Escola Politécnica

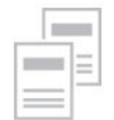

Relatórios da Escola Politécnica

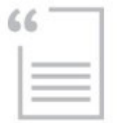

Revistas Politécnicas

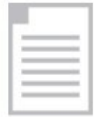

Atas da Congregaçāo

Programas de disciplinas

Figura 2 - Página inicial do repositório digital do Projeto Memória da Poli. Fonte: Memória da Poli, 2016.

No que diz respeito ao desenvolvimento da plataforma, os dois projetos são similares, com uma diferença em termos de escala: enquanto o primeiro contém cerca de 2000 arquivos, totalizando mais de 15000 páginas digitalizadas e de natureza heterogênea em termos de tamanho e conteúdo, o segundo é padronizado em termos de tipos de documentos, tratando-se basicamente de folhas A4 contendo texto, e é composto por 156 documentos. 


\section{(2) Bibliocanto?}

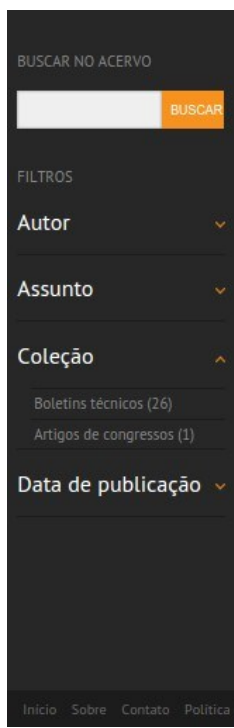

concreto

27 resultados

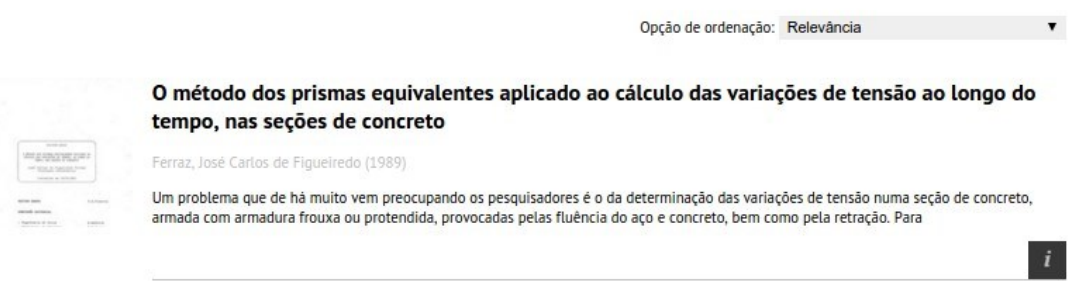

Sugestōes à norma, em discussāo, sobre "Projeto de estrutura de concreto protendido", NBR7197 (NB-116)

Neste trabalho, discute-se sobre a norma NBR-7197 (NB-116)

Figura 3 - Resultado da busca "concreto" no repositório digital do PEF-USP. Fonte: PEF, 2016.

As diferenças na natureza do material (principalmente a fragilidade dos documentos do primeiro projeto) geraram uma necessidade de desenvolvimento de procedimentos operacionais distintos. Enquanto no primeiro projeto pensou-se no sistema de digitalização, processamento, catalogação e disponibilização ao usuário final como um serviço (com todas as características de projetos de produção de serviços), no caso do segundo, pensou-se no sistema como um projeto de produção de um produto. Isto implica em processos mais rápidos no segundo caso devido à padronização das tarefas. Neste caso, como as folhas possuíam um tamanho padrão, foi utilizado um escâner multifuncional, eliminando a necessidade de processamento das imagens.

No caso do primeiro projeto, isto não foi possível, e um escâner planetário precisou ser adquirido. Em termos operacionais, isso aumentou o tempo total de digitalização em processamento de cerca de 15 minutos para um livro de 100 páginas para mais de 1 hora e 30 minutos, na média. 0 desvio padrão 


\section{Bibliocanto}

devido à heterogeneidade do material também aumentou consideravelmente, tornando muito difícil a predição de tempo de digitalização do material antes de uma verificação de seu estado.

No projeto Memória da Poli, os principais problemas observados podem ser separados em duas categorias: digitalização e catalogação. Na primeira, estes se relacionaram a: calibração do escâner, problemas com a câmera utilizada e problemas relacionados ao software de captura de imagens. Estes foram tratados no início do projeto, sendo resolvidos nos primeiros meses.

Os problemas relacionados à catalogação se relacionaram principalmente à descrição e análise dos metadados dos documentos em si, principalmente como realizar a equivalência entre os campos de metadados do Dublin Core (WEIBEL, 1997) (utilizado como padrão pelo software DSpace) e o padrão utilizado pela biblioteca. Neste caso específico, utilizou-se o MARC21 (LIBRARY OF CONGRESS, 1999). Para a resolução destes problemas, foi necessário utilizar uma tabela de equivalência de campos de metadados. Esta foi utilizada posteriormente em todas as implementações da plataforma. Parte desta tabela encontra-se no Quadro 1. 


\section{Bibliocantos}

116

\begin{tabular}{|l|l|}
\hline Campo de metadado no MARC21 & Campo de metadado no Dublin Core \\
\hline 245 title proper statement & Title (dc.title) \\
210 abbreviated title & \\
222 key title & \\
242 collective uniform title & \\
246 varying form of title & \\
247 former title & \\
\hline 520 summary note & Description (dc.description) \\
\hline Characteristics - general information (r) & Type (dc.type) \\
006 books & \\
006 computer files/electronic resources & \\
006 maps & \\
006 mixed materials & \\
006 music & \\
006 continuing resources & \\
006 visual materials & Contributors (dc.contributor) \\
\hline 100 main entry personal name \\
110 main entry corporate name \\
111 main entry meeting name \\
130 main entry uniform title & \\
\hline
\end{tabular}

Quadro 1 - Parte da tabela de equivalência entre MARC21 e Dublin Core

Fonte: CHUDAMANI, NAGARATHNA, 2006.

\subsection{IMPLEMENTAÇÃO EM ARQUIVOS HISTÓRICOS}

Os projetos de implementação da plataforma Papaya em arquivos históricos se deu após o término das implementações nas bibliotecas, citadas anteriormente, dentre outros projetos. Porém, ao contrário do que se pensava, o trabalho com arquivos é consideravelmente mais complexo do que com bibliotecas.

Dentre os principais problemas observados, pode-se citar:

- Dificuldade na interação entre as equipes de desenvolvimento da 


\section{Q Bibliocanto@}

plataforma e de gerenciadores de conteúdo, por estes utilizarem um vocabulário diferente dos bibliotecários;

- Heterogeneidade dos documentos, sendo que não existe um padrão. Os arquivos podem conter desde fotos $3 \times 4$ em preto e branco a documentos de grandes dimensões coloridos, transparências, papéis frágeis, além de documentos que estão fora dos tamanhos padrão de folhas. Isto dificulta não apenas os procedimentos operacionais, como também a forma de visualização dos documentos na plataforma, demandando outros visualizadores de imagens que permitam o carregamento da imagem por partes (devido a seu grande tamanho) e um zoom mais preciso;

- Dificuldade na realização da equivalência entre campos de metadados e os padrões utilizados nos arquivos;

- Caráter mais conservador por parte dos arquivos em relação às bibliotecas, devido a uma menor ocorrência de repositórios digitais de arquivos históricos no país;

- Fragilidade dos documentos, levando a um maior cuidado nos procedimentos operacionais de deslocamento e digitalização do material.

A plataforma foi implementada em duas instituições que possuem arquivos históricos durante os anos de 2015 e 2016: o Instituto Hercule Florence (IHF, 2016) e o Instituto do Patrimônio Histórico e Artístico Nacional (IPHAN, 2016). A Figura 4 contém a visualização de um documento do primeiro projeto, e a Figura 5 contém a visualização de um documento do segundo projeto. 


\section{3ibliocantos?}

118
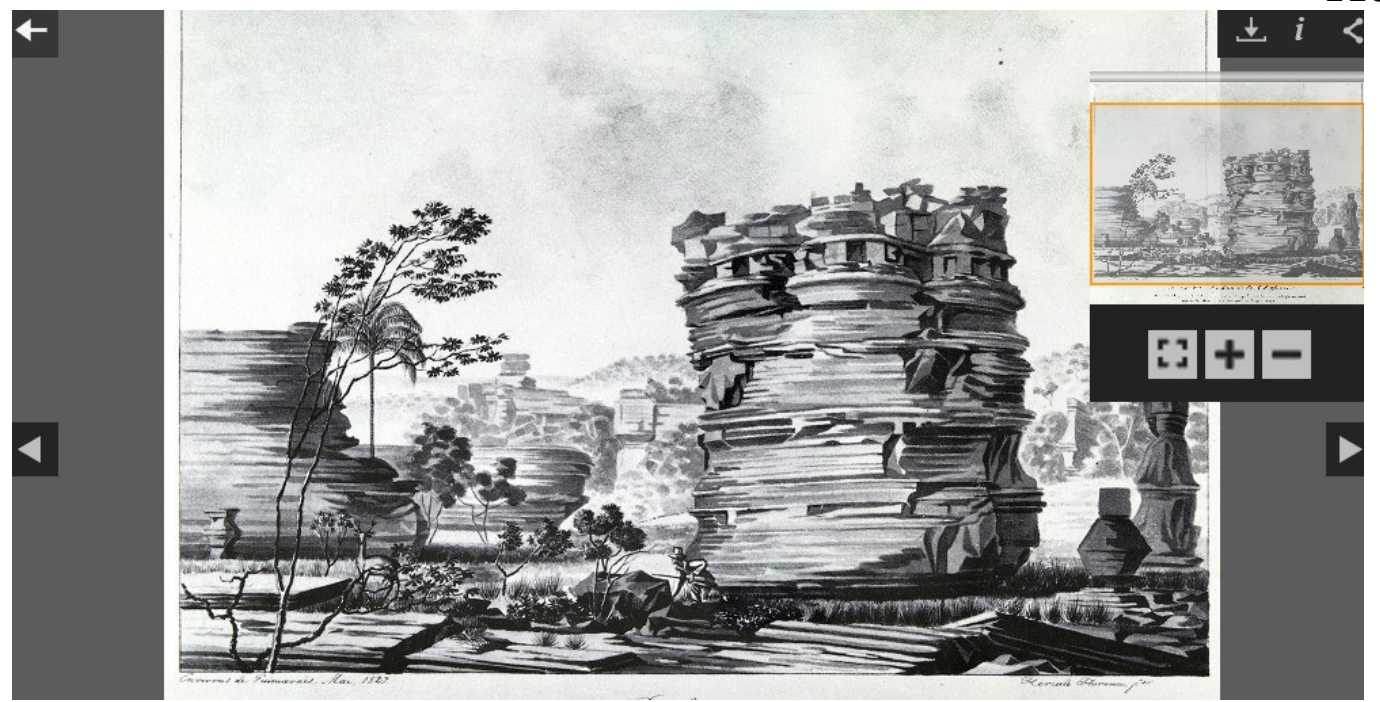

Figura 4 - Visualização de documento no repositório digital do IHF.

Fonte: IHF, 2016.

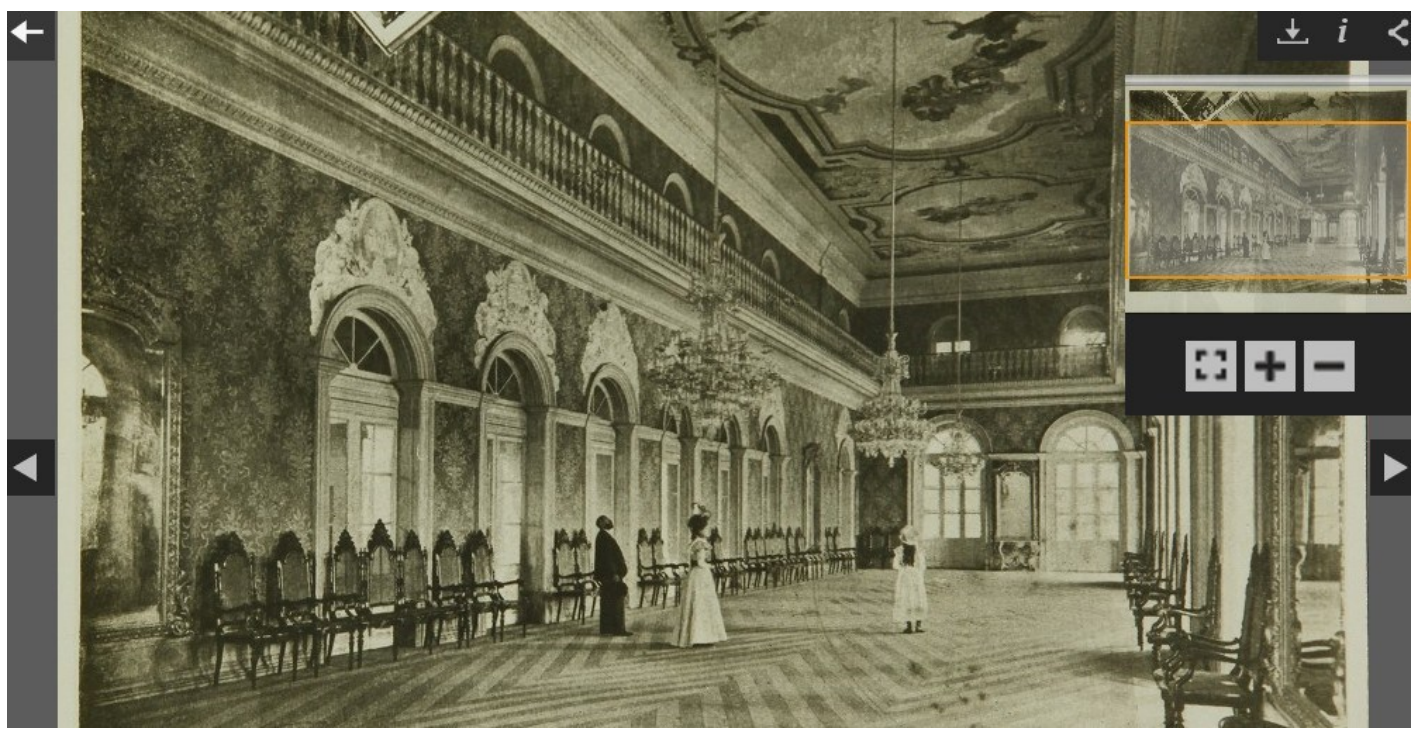

Figura 5 - Visualização de documento no repositório digital do IPHAN.

Fonte: IPHAN, 2016.

Como o acervo do IHF é majoritariamente composto por fotografias em tamanhos padronizados, matérias de jornais e documentos textuais, grande parte dos procedimentos utilizados nos projetos anteriores de 


\section{Q Bibliocanto@}

bibliotecas puderam ser reaproveitados. Este contém cerca de 300 documentos. Porém, este acervo apresentou alguns problemas particulares, principalmente relacionados à necessidade de visualização de imagens de alta qualidade no navegador do usuário final. Devido à esta razão, estudos de compressão de imagens e de velocidade na obtenção e carregamento das imagens foram conduzidos, e seus resultados estão sendo avaliados atualmente.

No caso do projeto de implementação do repositório digital do IPHAN, a interação entre as equipes se provou essencial. Por ser um projeto envolvendo uma equipe maior, diversas subequipes foram formadas: digitalização e processamento de imagens, descrição de documentos, preservação de documentos, análise de documentos, desenvolvimento de interface, e implementação. Um dos principais desafios foi a implementação de um repositório no qual o DSpace a Papaya se encontram instalados em um servidor, e o banco de dados encontra-se em outro servidor. Atualmente, este repositório conta com 8431 documentos.

Em termos de tempos de digitalização, ambos os projetos possuem tempos semelhantes ao projeto Memória da Poli, principalmente devido à heterogeneidade dos documentos. Os principais erros que ocorreram durante essas implementações foram relacionados principalmente à estruturação da hierarquia dos documentos no repositório digital, pois a definição das coleções e comunidades não é tão clara quanto no caso de bibliotecas, e na equivalência entre os sistemas de catalogação atualmente utilizados e o Dublin Core.

Durante este trabalho, foram expostos os principais requisitos para a implementação de repositórios digitais para bibliotecas e arquivos históricos, bem como da plataforma proposta para que esta pudesse ser realizada. 


\section{- Bibliocanto@}

120

Aspectos relacionados à implementação em si também foram abordados, fornecendo dois exemplos de uso de Plataforma Papaya para cada uma destas categorias.

As principais limitações encontradas durante a realização do trabalho podem ser divididas em duas frentes: relacionadas ao desenvolvimento da plataforma, principalmente no que diz respeito à seleção e adaptação dos softwares que foram utilizados para gerenciar o conteúdo e a visualização dos documentos; e relacionadas à implementação em si, principalmente relacionada à definição de processos operacionais adaptados para cada categoria de usuário.

Trabalhos futuros são relacionados à incorporação de novas funcionalidades na plataforma, como visualizadores para vídeo e 3D, o que permitiria fornecer uma ferramenta útil para museus e instituições com material destas naturezas, além de implementação em um maior número de bibliotecas e arquivos, desenvolvimento de procedimentos operacionais para o trabalho de digitalização em museus, e elaboração de uma metodologia para se definir qual é a compressão ideal para um determinado material, utilizando critérios quantitativos.

\section{CONSIDERAÇÕES FINAIS}

A digitalização de acervos memoriais é uma realidade no país. Porém, para melhorar a eficiência dos esforços nesta área, é necessário o desenvolvimento de ferramentas e procedimentos operacionais que permitam obter resultados de qualidade, evitando retrabalhos e a um baixo custo. Para que isto ocorra, é necessário conhecer os requisitos dos diferentes tipos de usuários, e desenvolver processos que permitam utilizar os seus recursos da melhor maneira possível. 


\section{Q Bibliocanto@}

121

Observou-se que a Papaya é uma plataforma que possibilita atender os requisitos tanto de arquivos quanto de bibliotecas. Também foi observado que o uso de softwares de código aberto é uma alternativa aos programas comerciais de código fechado, o que diminui os custos do projeto de digitalização.

Abstract: The library digitization process is very important as a tool to improve the reach of documents and their preservation. Implementing a digital repository able to accommodate a library collection is a complex task and it shows subtleties dependent on that collection's context and operational procedures. The Papaya Platform is a tool developed to help managing library collections. This paper analyzes four implementations of the platform, two on libraries and two on archives, highlighting their respective requisites and the decisions taken during the development phase.

Keywords: Library collections. Information access. Archives. Open source. Digital libraries.

\section{REFERÊNCIAS}

ADOBE. Adobe Flash Player. 2016. Disponível em: <http://www.adobe.com /products/flashplayer.html>. Acesso em: 20 jun. 2016.

ADORE DJATOKA. Adore Djatoka. 2013. Disponível em: $<$ https://sourceforge.net /projects/djatoka/>. Acesso em: 20 jun. 2016.

APACHE COCOON. Apache Cocoon. 2016. Disponível em: <http://cocoon. apache.org/>. Acesso em: 20 jun. 2016.

CHUDAMANI, K. S.; NAGARATHNA, H. C. Interoperability Between Dublin Core, Unimarc, Marc21, With AACR2R as the Standard Frameworks for Cataloguing in the Digital Environment. In: Proceedings of the 4th Convention Planner - 2006. INFLIBNET: Aizawl, India, p. 185-195, 2006. 


\section{(2) Bibliocantos}

COYLE, K. Mass digitization of books. The Journal of Academic

Librarianship, v. 32, n. 6, p. 641-645, 2006.

DSPACE. DSpace. 2016. Disponível em: <http://www.dspace.org/>. Acesso em: 20 jun. 2016.

EMBRAPA. Sistema Alice. 2016. Disponível em: <https://www.alice.cnptia. embrapa.br/>. Acesso em: 15 jun. 2016.

FIOCRUZ. Biblioteca Multimídia da FIOCRUZ. 2016. Disponível em:<http://www5. ensfiocruz.br/biblioteca/home/>. Acesso em: 15 jun. 2016.

INSTITUTO BRASILEIRO DE INFORMAÇÃO EM CIÊNCIA E TECNOLOGIA. Lista de repositórios brasileiros. 2016. Disponível em:

$<$ http://www.ibict.br/informacao-para-ciencia-tecnologia-einovacao\%20/repositorios-digitais/ repositorios-brasileiros>. Acesso em: 20 jun. 2016.

INSTITUTO HERCULE FLORENCE. Acervo digital do Instituto Hercule Florence. 2016. Disponível em: <http://search.ihf19.org.br:8080/xmlui/>. Acesso em: 15 jun. 2016.

INMETRO. Acervo digital INMETRO. 2016. Disponível em: <http://repositorios. inmetro.gov.br/>. Acesso em: 15 jun. 2016.

INTERNET ARCHIVE. BookReader. 2015. Disponível em: < https://openlibrary. org/ dev/docs/ bookreader>. Acesso em: 20 jun. 2016.

IIP IMAGE. IIPMooViewer. 2015. Disponível em:

$<$ http://iipimage.sourceforge.net/ documentation/iipmooviewer/>. Acesso em: 20 jun. 2016.

IPHAN. Rede de arquivos do IPHAN. 2016. Disponível em:

<http://acervodigital. iphan.gov.br/xmlui/>. Acesso em: 15 jun. 2016.

LESK, M. Why digital libraries? United Kingdom Office for Library and Information Networking, UKOLN, 1995.

LIBRARY OF CONGRESS. MARC 21 format for bibliographic data. 1999. Disponível em: <https://www.loc.gov/marc/bibliographic/>. Acesso em: 15 jun. 2016. 


\section{- Bibliocantoo}

LYNCH, C. A. Institutional Repositories: Essential Infrastructure for Scholarship in the Digital Age. ARL: A Bimonthly Report, p. 1-7, 2003.

MARTINS, A.; RODRIGUES, E.; NUNES, M. B. Repositórios de informação e ambientes de aprendizagem: Criação de espaços virtuais para a promoção da literacia e da responsabilidade social. Rede de Bibliotecas Escolares Newsletter, n. 3, p. 1-5, 2008.

MEMORIA DA POLI. Memória da Escola Politécnica da USP. 2016.

Disponível em: <http://memoria.poli.usp.br/>. Acesso em: 15 jun. 2016.

PEF. Acervo digital do PEF-USP. 2016. Disponível em: <http://acervodigital. pef.usp.br/>. Acesso em: 15 jun. 2016.

PHILLIPS, S.; GREEN, C.; MASLOV, A.; MIKAEL, A.; LEGGETT, J. Manakin: A New Face for DSpace. D-Lib Magazine, v. 14, n. 11/12, 2007.

PLATAFORMA PAPAYA. Plataforma Papaya. 2016. Disponível em: <http://plataformapapaya.org/>. Acessado em:20/06/2016.

TANSLEY, R. et al. The DSpace institutional digital repository system: current functionality. Proceedings of the 3rd ACM/IEEE-CS joint conference ongital libraries (JCDL '03). IEEE: Houston, USA, p. 87-97, 2003.

UNIVERSIDADE DE SÃO PAULO. Biblioteca Digital da Produção Intelectual da Universidade de São Paulo. 2016. Disponível em:<http://www.producao. usp.br/ >. Acesso em: 15jun. 2016.

WEIBEL, S. The Dublin Core: A Simple Content Description Model for Electronic Resources. Bul. Am. Soc. Info. Sci. Tech., v.24, p.9-11, 1997. 